\title{
Transversity quark distributions in a covariant quark-diquark model
}

\author{
I. C. Cloët,,${ }^{1, *}$ W. Bentz, ${ }^{2, \dagger}$ and A. W. Thomas ${ }^{3,4, \ddagger}$ \\ ${ }^{1}$ Physics Division, Argonne National Laboratory, Argonne, IL 60439-4843, U.S.A. \\ ${ }^{2}$ Department of Physics, School of Science, Tokai University, Hiratsuka-shi, Kanagawa 259-1292, Japan \\ ${ }^{3}$ Jefferson Lab, 12000 Jefferson Avenue, Newport News, VA 23606, U.S.A. \\ ${ }^{4}$ College of William and Mary, Williamsburg, VA 23187, U.S.A.
}

\begin{abstract}
Transversity quark light-cone momentum distributions are calculated for the nucleon. We utilize a modified Nambu-Jona-Lasinio model in which confinement is simulated by eliminating unphysical thresholds for nucleon decay into quarks. The nucleon bound state is obtained by solving the relativistic Faddeev equation in the quark-diquark approximation, where both scalar and axialvector diquark channels are included. Particular attention is paid to comparing our results with the recent experimental extraction of the transversity distributions by Anselmino et al. We also compare our transversity results with earlier spin-independent and helicity quark distributions calculated in the same approach.
\end{abstract}

PACS numbers: 13.60.Hb,12.38.Lg,11.80.Jy,12.39.Fe,12.39.Ki

\section{INTRODUCTION}

For perfectly collinear quarks, at leading twist, the nucleon has three independent quark distribution functions for each quark flavour: the unpolarized distributions, $q(x)$, the helicity distributions, $\Delta q(x)$, and the transversity distributions, $\Delta_{T} q(x)$. Knowledge of each distribution is of equal importance if one is to have a robust description of nucleon structure. The unpolarized and helicity distributions have been extensively studied, both experimentally and theoretically, for many years $[1,2]$. However interest in the transversity distributions is relatively recent [3].

The transversity distributions are associated with quark-nucleon forward Compton amplitudes, where both the quark and nucleon helicities are flipped. Hence these distributions are chiral-odd. The electroweak and strong interactions conserve chirality, so the transversity distributions must couple to another chiral-odd quantity in scattering cross-sections. This is not possible in inclusive deep inelastic scattering (DIS) [3]. However, for certain semi-inclusive DIS [3-5] and Drell-Yan processes [3, 6-8] the transversity distributions do appear at leading twist in the cross-section. For semi-inclusive DIS the transverse quark distributions couple to particular chiral-odd fragmentation functions (Collins functions), whereas in Drell-Yan dilepton production they appear with either a transverse quark or anti-quark distribution from the partner hadron.

The transversity distributions are particularly interesting for several reasons, for example:

- the moments of the transversity valence distributions are related to the nucleon tensor charge [9];

- the quark bilinears associated with transversity are odd under charge conjugation and hence the quarkanti-quark sea does not contribute [9];

- helicity conservation at the quark-gluon vertex prevents mixing between the quark and gluon transversity distributions under QCD evolution $[7,10,11]$

- gluon transversity distributions, $\Delta_{T} g(x)$, only exist for targets with $J \geqslant 1$, because measurement of a gluon transversity distribution requires that the target change helicity by two units of angular momentum and this is not possible for spin- $\frac{1}{2}$ targets $[3,4,7]$.

These results imply that the transversity distributions are valence quark dominated and evolve as non-singlets under DGLAP evolution, where the angular momentum generated by the DGLAP kernels is not shared between the quark and gluon sectors. These features of the transversity distributions make them particularly amenable to a quark model treatment.

In this paper we calculate the transversity distributions for the proton using a Nambu-Jona-Lasinio model $[12,13]$. This model is attractive because it is covariant and has a transparent description of spontaneous chiral symmetry breaking. Confinement - in the sense that there exists no threshold for nucleon decay into quarks - is also implemented via the regularization procedure $[14,15]$. We construct the nucleon as a bound state solution of the relativistic Faddeev equation [16], in the quark-diquark approximation $[17,18]$, where both scalar and axial-vector diquark channels are included. We compare our transversity results to spin-independent and helicity quark distributions calculated in the same approach [19]. Particular attention is also paid to a comparison of our transversity results with the recent, and to date the only, experimental extraction of the transversity distributions by Anselmino et al., presented in Ref. [20].

\section{TRANSVERSITY QUARK DISTRIBUTIONS}

The transversity distributions render a probability interpretation analogous to the other two leading twist distributions. In a transversely polarized hadron they 
represent the number density of quarks in an eigenstate of the transverse Pauli-Lubanski operator, $\$_{\perp} \gamma_{5}$, with eigenvalue $+\frac{1}{2}$, minus the number density of quarks with eigenvalue $-\frac{1}{2},[8]$ that is

$$
\Delta_{T} q(x)=q_{\uparrow}(x)-q_{\downarrow}(x) .
$$

In an helicity basis the helicity distributions are expressed as

$$
\Delta q(x)=q_{+}(x)-q_{-}(x),
$$

where $q_{+}(x)$ is the number density of quarks with helicity parallel to the hadron helicity and $q_{-}(x)$ is the quark number density with helicity anti-aligned. The spin-independent distributions in each basis are given by

$$
q(x)=q_{\uparrow}(x)+q_{\downarrow}(x)=q_{+}(x)+q_{-}(x) .
$$

The leading twist quark distribution functions are defined by light-cone Fourier transforms of connected matrix elements of particular quark field bilinears. For example, the twist-2 transversity distribution is defined by

$$
\begin{aligned}
\Delta_{T} q(x)=p^{+} \int \frac{d \xi^{-}}{2 \pi} e^{i x p^{+} \xi^{-}} & \\
& \left\langle p, s\left|\bar{\psi}_{q}(0) \gamma^{+} \gamma^{1} \gamma_{5} \psi_{q}\left(\xi^{-}\right)\right| p, s\right\rangle_{c},
\end{aligned}
$$

where $\psi_{q}$ is a quark field of flavour $q$ and $x$ is the Bjorken scaling variable. ${ }^{1}$ In Eq. (4) the target polarization is in the $x$-direction, with the $z$-direction defined by the photon 3-momentum.

The evaluation of the quark distributions is facilitated by expressing Eq. (4) in the form [21, 22]

$$
\Delta_{T} q(x)=-i \int \frac{d^{4} k}{(2 \pi)^{4}} \delta\left(x-\frac{k^{+}}{p^{+}}\right) \operatorname{Tr}\left[\gamma^{+} \gamma^{1} \gamma_{5} M(p, k)\right]
$$

where $M(p, k)$ is the quark two-point function in a nucleon. The quark distributions can then be expressed in terms of Feynman diagrams for any model where the nucleon is represented by a bound state of quarks. The diagrams we consider are given in Fig. 1. In our pure valence quark model there should also be a third diagram, the so-called quark exchange term [17], however this diagram does not contribute within the static approximation used here $[16,18]$.

In the Feynman diagrams of Fig. 1 the single line represents a constituent quark propagator and the double line a diquark $t$-matrix. The diagram on the left is referred to as the quark diagram and on the right we have

\footnotetext{
1 The formal expressions for the spin-independent and helicity distributions can be obtained from Eq. (4) via the operator replacements $\gamma^{+} \gamma^{1} \gamma_{5} \rightarrow \gamma^{+}$and $\gamma^{+} \gamma^{1} \gamma_{5} \rightarrow \gamma^{+} \gamma_{5}$, respectively. The nucleon state is normalized according to the non-covariant lightcone normalization, namely $\left\langle p, s\left|\bar{\psi}_{u} \gamma^{+} \psi_{u}+\bar{\psi}_{d} \gamma^{+} \psi_{d}\right| p, s\right\rangle_{c}=3$.
}

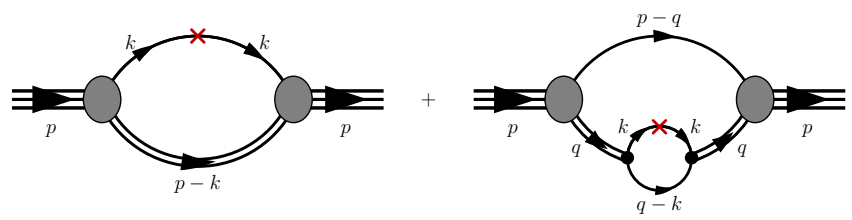

FIG. 1: Feynman diagrams representing the transversity quark distributions in the nucleon, needed in the evaluation of Eq. (5). The single line represents the quark propagator and the double line the diquark $t$-matrix. The shaded oval denotes the quark-diquark vertex function and the operator insertion has the form $\gamma^{+} \gamma^{1} \gamma_{5} \delta\left(x-\frac{k^{+}}{p^{+}}\right) \frac{1}{2}\left(1 \pm \tau_{z}\right)$.

the diquark diagram, where we include both scalar and axial-vector diquarks. Separating the isospin coefficients, the $u$ - and $d$-quark transversity distributions can be expressed as

$$
\begin{aligned}
\Delta_{T} u_{v}(x) & =\Delta_{T} f_{q / N}^{s}(x) \\
+\frac{1}{2} \Delta_{T} f_{q(D) / N}^{s}(x)+\frac{1}{3} \Delta_{T} f_{q / N}^{a}(x) & \\
+ & \frac{5}{6} \Delta_{T} f_{q(D) / N}^{a}(x)+\frac{1}{2 \sqrt{3}} \Delta_{T} f_{q(D) / N}^{m}(x), \\
\Delta_{T} d_{v}(x) & =\frac{1}{2} \Delta_{T} f_{q(D) / N}^{s}(x)+\frac{2}{3} \Delta_{T} f_{q / N}^{a}(x) \\
+ & \frac{1}{6} \Delta_{T} f_{q(D) / N}^{a}(x)-\frac{1}{2 \sqrt{3}} \Delta_{T} f_{q(D) / N}^{m}(x),
\end{aligned}
$$

where each term represents a particular Feynman diagram in Fig. 1. The superscripts $s, a$ and $m$ refer to the scalar, axial-vector or mixing terms, respectively. The subscript $q / N$ implies a quark diagram and $q(D) / N$ a diquark diagram. Because the scalar diquark has spin zero, we have $\Delta_{T} f_{q(D) / N}^{s}(x)=0$ and hence the transversity of the $d$-quark arises exclusively from the axial-vector and the mixing terms.

Importantly, in this covariant framework, the Ward identities corresponding to number and momentum conservation are satisfied, guaranteeing the validity of the baryon number and momentum sum rules [23, 24] from the outset.

\section{THE NUCLEON IN THE NJL MODEL}

The NJL model which we employ to determine the transversity distributions is discussed in Ref. [19], where it was used to determine the spin-independent and helicity quark distribution functions. Therefore we give only a brief outline of the model in this section and refer the reader to Ref. [19] for further details.

We represent the nucleon as a quark-diquark bound state, where both the scalar and axial-vector diquark channels are included. The nucleon $t$-matrix is obtained as the solution of the relativistic Faddeev equation

$$
T=Z+Z \Pi_{N} T=Z+T \Pi_{N} Z .
$$


In the static approximation the quark exchange kernel, $Z$, becomes [16]

$$
Z=\frac{3}{M}\left(\begin{array}{cc}
1 & \sqrt{3} \gamma^{\mu} \gamma_{5} \\
\sqrt{3} \gamma_{5} \gamma^{\mu} & -\gamma^{\mu} \gamma^{\mu^{\prime}}
\end{array}\right)
$$

where $M$ is the constituent quark mass. With this approximation, $\Pi_{N}$ of Eq. (8) effectively becomes the quark-diquark bubble graph, given by

$$
\Pi_{N}(p)=\int \frac{d^{4} k}{(2 \pi)^{4}} \tau(p-k) S(k)
$$

where

$$
\tau(q)=\left(\begin{array}{cc}
\tau_{s}(q) & 0 \\
0 & \tau_{a}^{\mu \nu}(q)
\end{array}\right) .
$$

The quantities $\tau_{s}(q)$ and $\tau_{a}^{\mu \nu}(q)$ are the solutions to the NJL scalar and axial-vector diquark Bethe-Salpeter equations, respectively [16, 25, 26], and $S(k)$ in Eq. (10) is the usual fermion propagator for a constituent quark.

In the non-covariant light-cone normalization used already in Eq. (4), the quark-diquark vertex function, $\Gamma_{N}$, is defined by the behaviour of $T$ near the pole

$$
T \stackrel{p_{+} \rightarrow \varepsilon_{p}}{\longrightarrow} \frac{\Gamma_{N} \bar{\Gamma}_{N}}{p_{+}-\varepsilon_{p}}
$$

where $\varepsilon_{p}=\frac{M_{N}^{2}}{2 p_{-}}$is the nucleon's light-cone energy. The vertex functions satisfy homogeneous Faddeev equations of the form

$$
\Gamma_{N}=Z \Pi_{N} \Gamma_{N}, \quad \text { and } \quad \bar{\Gamma}_{N}=\bar{\Gamma}_{N} \Pi_{N} Z .
$$

As the NJL model is non-renormalizable, a regularization prescription must be specified to fully define the theory. We choose the proper-time regularization scheme $[14,15,27-29]$, where loop integrals of products of propagators are evaluated by introducing Feynman parameters, Wick rotating and making the denominator replacement

$$
\frac{1}{X^{n}} \longrightarrow \frac{1}{(n-1) !} \int_{1 /\left(\Lambda_{U V}\right)^{2}}^{1 /\left(\Lambda_{I R}\right)^{2}} d \tau \tau^{n-1} e^{-\tau X},
$$

where $\Lambda_{I R}$ and $\Lambda_{U V}$ are, respectively, the infrared and ultraviolet cutoffs. The former has the effect of eliminating unphysical thresholds for hadron decay into quarks, hence simulating an important aspect of quark confinement [15]. The proper-time scheme also preserves the gauge invariance and Poincaré covariance of the theory.

\section{RESULTS}

The six parameters of the model are: the regularization parameters, $\Lambda_{I R}$ and $\Lambda_{U V}$, the current quark mass, $m$, and three quark-quark coupling constants $G_{\pi}, G_{s}$ and

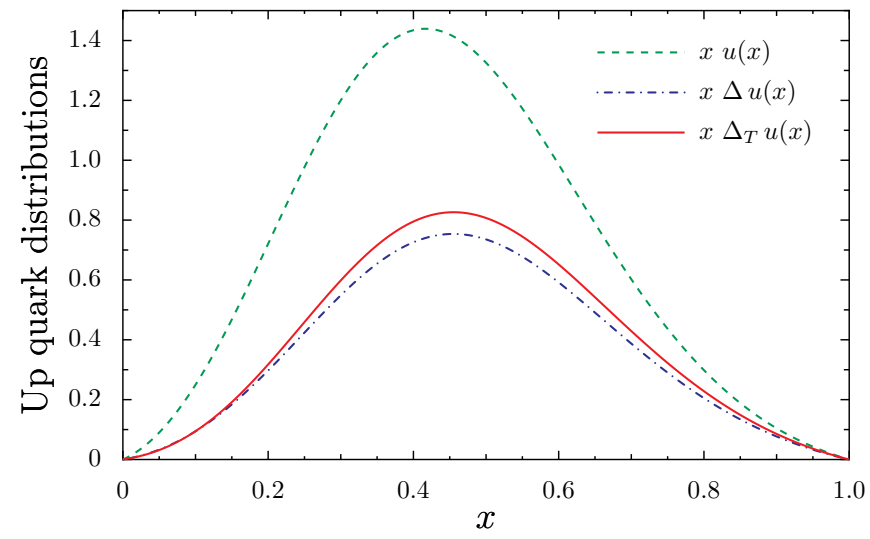

FIG. 2: Model results for the triplet of twist-2 valence up quark distributions, at $Q_{0}^{2}=0.16 \mathrm{GeV}^{2}$. The spinindependent and helicity distributions are taken from earlier work presented in Ref. [19].

$G_{a}$ [19]. The infrared or confinement scale is expected to be of order $\Lambda_{\mathrm{QCD}}$ and we choose $\Lambda_{I R}=240 \mathrm{MeV}$, however our results exhibit only a minor dependence on $\Lambda_{I R}$ when it is varied between $200-300 \mathrm{MeV}$. The parameters $m, \Lambda_{U V}$ and the $q \bar{q}$ coupling in the pion channel, $G_{\pi}$, are determined by requiring $M=400 \mathrm{MeV}$ via the gap equation, $f_{\pi}=93 \mathrm{MeV}$ from the one loop pion decay diagram and $m_{\pi}=140 \mathrm{MeV}$ from the pole of the $q \bar{q} t$-matrix in the pion channel. This gives $m=16.4 \mathrm{MeV}, \Lambda_{U V}=645 \mathrm{MeV}$ and $G_{\pi}=19.04 \mathrm{GeV}^{-2}$. The $q q$ couplings in the scalar, $G_{s}$, and axial-vector, $G_{a}$, diquark channels are determined by reproducing the nucleon mass, $M_{N}=940 \mathrm{MeV}$, as the solution of Eq. (13) and satisfying the Bjorken sum rule within our model, with $g_{A}=1.267$. We obtain $G_{s}=7.49 \mathrm{GeV}^{-2}$ and $G_{a}=2.80 \mathrm{GeV}^{-2}$. With these model parameters the diquark masses are $M_{s}=687 \mathrm{MeV}$ and $M_{a}=1027 \mathrm{MeV}$.

Inherent in most model determinations of the quark distributions is the absence of an explicit $Q^{2}$ scale. Consequently the model scale, $Q_{0}^{2}$, must be determined via comparison with empirical results. We do this by optimizing $Q_{0}^{2}$ such that the spin-independent valence up quark distribution, $u_{v}(x) \equiv u(x)-\bar{u}(x)$, best reproduces the empirical parametrization after NLO $Q^{2}$ evolution. $^{2}$ We find $Q_{0}^{2}=0.16 \mathrm{GeV}^{2}$ [19], which is typical of valence dominated models $[23,26,30]$.

In Figs. 2 and 3 we illustrate our results for the transversity valence $u$ - and $d$-quark distributions, respectively. Included in these figures are our results for the spin-independent and helicity valence distributions taken from Ref. [19]. We find a transversity $u$-quark distribution slightly larger than the corresponding helicity dis-

\footnotetext{
2 The DGLAP evolution of our results for the three twist-2 quark distributions is performed using the appropriate computer program from Refs. [31-33]. For the DGLAP parameters we choose $N_{f}=3$ and $\Lambda_{\mathrm{QCD}}=250 \mathrm{MeV}$.
} 


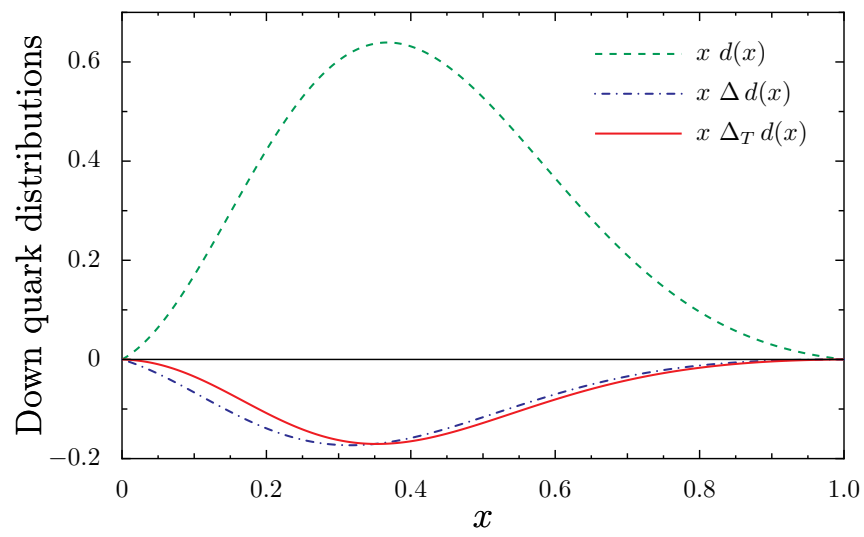

FIG. 3: Model results for the triplet of twist-2 valence down quark distributions, at $Q_{0}^{2}=0.16 \mathrm{GeV}^{2}$. The spinindependent and helicity results are taken from earlier work presented in Ref. [19].

tribution for all $x$. The transversity and helicity $d$-quark distributions are very similar for $x \gtrsim 0.35$, however at smaller $x$ the transversity distribution is noticeably suppressed. The difference between the transversity and helicity valence distributions results purely from relativistic effects, since in the non-relativistic limit, where boosts and rotations commute, there can be no preferential polarization direction and therefore the helicity and transversity distributions must be equal. This is true only at the model scale as the helicity and transversity distributions evolve differently under DGLAP evolution.

The first experimental extraction of the transversity distributions was achieved only recently, and is published in Ref. [20]. The authors combined semi-inclusive DIS data from HERMES and COMPASS with $e^{+} e^{-}$ annihilation data from BELLE to simultaneously extract the transversity distributions and Collins functions. Their results for the transversity $u$ - and $d$-quark distributions at $Q^{2}=2.4 \mathrm{GeV}^{2}$ are presented in Fig. 4 as the shaded regions, which represent a one-sigma confidence interval. Included in this figure are our valence results at $Q^{2}=2.4 \mathrm{GeV}^{2}$ and the empirical Soffer bound at the same scale obtained from an evolution of the GRV parametrizations given in Refs. [34, 35]. A direct comparison between our results and those of Ref. [20] should be valid for $x>0.2$ where transversity anti-quark distributions are expected to be small. We find that our results lie slightly outside the one-sigma error bounds of the empirical parametrizations for $x \gtrsim 0.3$. On the same figure we illustrate our results for the helicity distributions at $Q^{2}=2.4 \mathrm{GeV}^{2}$. At this scale our helicity and transversity distributions remain very similar for $x \gtrsim 0.4$, however the differing $Q^{2}$ evolution has resulted in a substantial suppression for the transversity distributions at smaller $x$, when compared with the results at the model scale. The helicity distributions given in Fig. 4 are in excellent agreement with the empirical parametrizations [19].

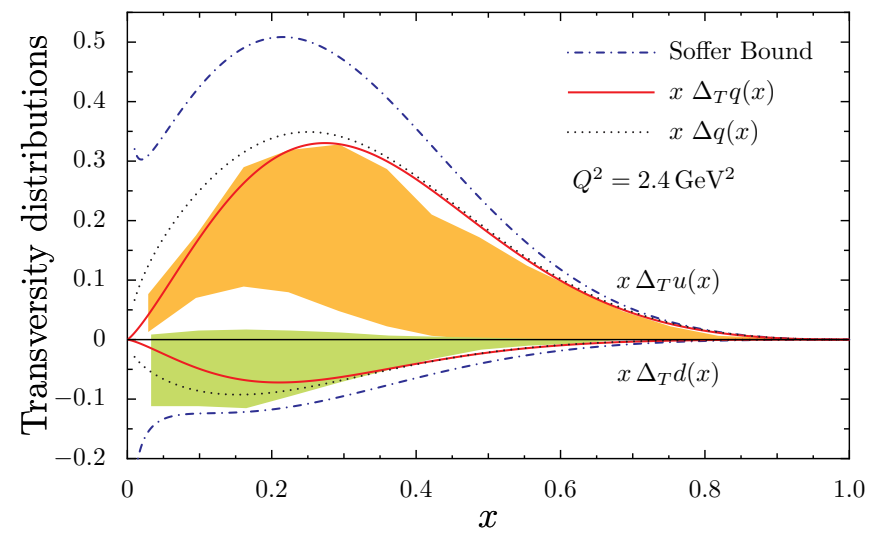

FIG. 4: The shaded areas are the empirical results of Ref. [20], with a one-sigma confidence interval. The dot-dashed line is the GRV Soffer bound [34, 35] and the solid lines are our results for the transversity distributions. The dotted curves are our helicity distributions taken from Ref. [19]. All results are at $Q^{2}=2.4 \mathrm{GeV}^{2}$.

The first moments of the transversity valence distributions are related to the nucleon's isovector tensor charge, $g_{T}$, via $[8]$

$$
\int_{0}^{1} d x\left[\Delta_{T} u_{v}\left(x, Q^{2}\right)-\Delta_{T} d_{v}\left(x, Q^{2}\right)\right]=g_{T}\left(Q^{2}\right) .
$$

The nucleon's isoscalar tensor charge, $g_{T}^{0}$, is defined as the sum of the valence transversity moments. For these moments we obtain $\Delta_{T} u_{v}=1.04$ and $\Delta_{T} d_{v}=-0.24$, giving a nucleon isovector tensor charge of $g_{T}=1.28$ and a isoscalar charge of $g_{T}^{0}=0.80$, at $Q^{2}=0.16 \mathrm{GeV}^{2}$. At the GRV scale of $Q^{2}=0.4 \mathrm{GeV}^{2}$ we obtain $\Delta_{T} u_{v}=0.69$, $\Delta_{T} d_{v}=-0.16, g_{T}=0.85$ and $g_{T}^{0}=0.53 .^{3}$ It is well known that the first moments of the helicity distributions give the quark spin content of the nucleon. However, the first moments of the transversity distributions are not equivalent to the quark spin in the transverse direction, since the expectation value of $\gamma^{1} \gamma_{5}$ is not equal to that of $\Sigma_{\perp}=\gamma^{0} \gamma^{1} \gamma_{5}$. That is, the isoscalar tensor charge cannot be interpreted as a transverse spin sum $[8,9]$.

The paper of Anselmino et al. does not give explicit values for the transversity moments, however if we integrate their parametrizations and accurately determine the errors from Fig. 7 in Ref. [20], we find $\Delta_{T} u=0.46_{-0.28}^{+0.36}$ and $\Delta_{T} d=-0.19_{-0.23}^{+0.30}$ at $Q^{2}=0.4 \mathrm{GeV}^{2} .{ }^{4}$ Using these mo-

3 These values are obtained using the NLO result [3]

$$
\begin{aligned}
\Delta_{T} q\left(Q^{2}\right)= & {\left[\frac{\alpha_{s}\left(Q^{2}\right)}{\alpha_{s}\left(Q_{0}^{2}\right)}\right]^{\frac{4}{27}} } \\
& {\left[1-\frac{337}{486 \pi}\left[\alpha_{s}\left(Q_{0}^{2}\right)-\alpha_{s}\left(Q^{2}\right)\right]\right] \Delta_{T} q\left(Q_{0}^{2}\right) . }
\end{aligned}
$$

4 These values are obtained from the FIT-I results given in Ta- 


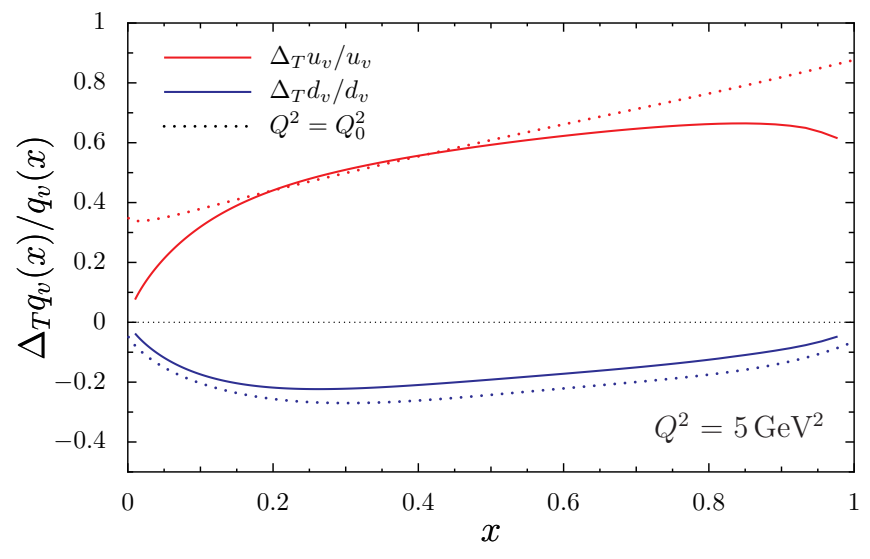

FIG. 5: Quark distribution ratios, in each case the dotted line is the result before QCD evolution and the solid line is the result for $Q^{2}=5 \mathrm{GeV}^{2}$.

ments their tensor charges are $g_{T} \sim 0.65$ and $g_{T}^{0} \sim 0.27$, assuming $\Delta_{T} \bar{u}-\Delta_{T} \bar{d} \simeq 0$.

The similarity between the transversity and helicity distributions, which we illustrate in Fig. 4, is a common theme for model calculations - see Refs. [8, 36-41]. This should come as no surprise since the constituent quark masses are typically rather large, $350-450 \mathrm{MeV}$, in these calculations and therefore enormous relativistic effects would be unexpected. For the moment, we have no more than a hint that this may disagree with the experimental extraction of Anselmino et al., who find a $u$-quark transversity distribution that may in fact be far smaller than its helicity counterpart. This difference is emphasized if we study the moments. The empirical values for the helicity moments are $\Delta u=0.851 \pm 0.075$ and $\Delta d=$ $-0.415 \pm 0.124$ [42], for the valence distributions the results are $\Delta u_{v}=0.926 \pm 0.071$ and $\Delta d_{v}=-0.341 \pm 0.123$ [42]. Therefore $\Delta_{T} u$ extracted from the fit of Ref. [20] is potentially far smaller than the $u$-quark helicity moment. If this difference survives it would indicate a difficulty for relativistic model approaches based on the concept of a constituent quark mass.

Within our NJL model calculation we find $\Delta u_{v}=0.97$ and $\Delta d_{v}=-0.30,{ }^{5}$ which differ only slightly from the transversity results given earlier. The nine models discussed in the transversity review by Barone et al. [3] all find $\Delta_{T} u>\Delta u$ and $\Delta_{T} d \sim \Delta d$ at the model scale. This consensus amongst the various model approaches,

ble I of Ref. [20]. The FIT-II results give even smaller moments, namely $\Delta_{T} u=0.40$ and $\Delta_{T} d=-0.16$. The errors quoted in the text are first obtained at $Q^{2}=2.4 \mathrm{GeV}^{2}$, then the NLO result expressed in Eq. (16) is used to devolve to the parametrization scale, $Q^{2}=0.4 \mathrm{GeV}^{2}$. We find that this procedure works very well for the central value, which can be determined at both $Q^{2}$ scales.

${ }^{5}$ Note, the helicity moments given here differ slightly from those in Ref. [19] as in this work the infrared cutoff is slightly different.

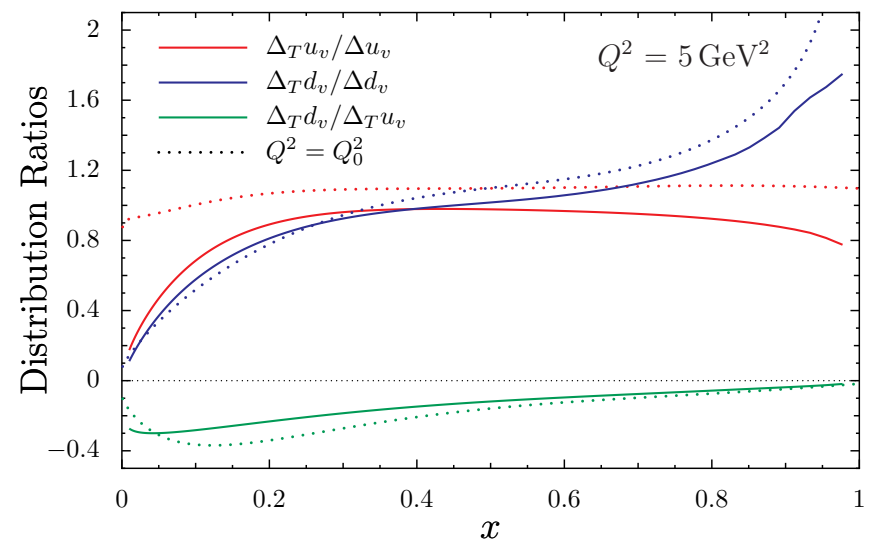

FIG. 6: Quark distribution ratios, in each case the dotted line is the result before QCD evolution and the solid line is the result for $Q^{2}=5 \mathrm{GeV}^{2}$.

together with our results, contrasted with the potentially small empirical $u$-quark transversity parametrization given in Ref. [20], presents an interesting problem. We therefore eagerly await new experiment data so that the empirical transversity distributions can be better constrained.

The Soffer inequality [44], $q(x)+\Delta q(x) \geqslant 2\left|\Delta_{T} q(x)\right|$, and the more familiar positivity conditions shed little light on the size of the $u$-quark transversity distribution, simply that $\left|\Delta_{T} u(x)\right| \leqslant u(x)$. However, the differing signs of the spin-averaged and helicity $d$-quark distributions implies that for all values of $x$ where $\Delta d(x) \leqslant 0$ the transversity $d$-quark distribution must satisfy $\left|\Delta_{T} d(x)\right| \leqslant$ $\frac{1}{2} d(x)$. For the $d$-quark moments the Soffer inequality implies $\left|\Delta_{T} d\right| \leqslant \frac{1}{2}$, provided $\Delta d \leqslant 0$. In fact, if $\Delta d \leqslant-\frac{1}{3}$, which appears true empirically [35, 42, 43], then the transversity moment must satisfy $\left|\Delta_{T} d\right| \leqslant \frac{1}{3}$. Our results, presented in Figs. 2 and 3, satisfy all positivity constraints, including the Soffer bound.

The relative importance of each diagram in Fig. 1 to the quark distributions is easily illustrated if we consider their contributions to the transversity moments. For the $u$-quark distributions we have $\Delta_{T} f_{u / N}^{s}=0.75$, $\Delta_{T} f_{u / N}^{a}=-0.016, \Delta_{T} f_{u(D) / N}^{s}=0, \Delta_{T} f_{u(D) / N}^{a}=0.090$ and $\Delta_{T} f_{u(D) / N}^{m}=0.22$, where the isospin factors in Eq. (6) have been incorporated into the numerical values. Results for the $d$-quark are obtained by dividing out the $u$-quark isospin factors and multiplying by the $d$-quark factors from Eq. (7). The dominant terms for $\Delta_{T} u(x)$ are clearly the scalar-quark and diquark-mixing diagrams. The strength of $d$-quark distribution arises almost exclusively from the diquark-mixing term. Similar results are also obtained for the helicity distributions [19]. This clearly illustrates the importance of including axialvector diquark correlations in any quark-diquark picture of the nucleon.

In Figs. 5 and 6 we illustrate various ratios of the transversity distributions with the unpolarized and helic- 
ity distributions. In each case the dotted line is the result at the model scale, $Q^{2}=Q_{0}^{2}$, and the solid line is the ratio evolved to $Q^{2}=5 \mathrm{GeV}^{2}$. In Fig. 5 we present our results for the single flavour ratios of transversity to unpolarized quark distributions. Both the up and down quark ratios are finite in the $x \rightarrow 1$ limit at the model scale. However, as all transversity moments decrease with increasing $Q^{2}$, we expect the ratios to decrease after $\mathrm{QCD}$ evolution, which is evident for the $Q^{2}=5 \mathrm{GeV}^{2}$ results. ${ }^{6}$

Ratios of transversity to helicity distributions are presented in Fig. 6. In the large $x$ limit we find that the $u$ quark ratio approaches 1.1 and the $d$-quark ratio becomes quite large. The $d$-quark result is surprising. It may be an inherent feature of the model or possibly a consequence of the static approximation. The quark exchange diagram, absent in this approximation, contributes dominantly in the scalar diquark channel, where a $d$-quark must be exchanged. Therefore, including this contribution may significantly alter the ratio $\Delta_{T} d(x) / \Delta d(x)$ at large $x$. This possibility illustrates the importance of going beyond the static approximation in future work. The final result illustrated in Fig. 6 is the mixed flavour transversity ratio, $\Delta_{T} d_{v}(x) / \Delta_{T} u_{v}(x)$, which is small for all $x$ and tends to zero as $x$ approaches one.

To aid comparison between our calculations and future investigations of the transversity distributions, we provide a parametrization of our $u$ - and $d$-quark valence distributions at the GRV scale, $Q^{2}=0.4 \mathrm{GeV}^{2}$. The general form of the parametrization is

$$
x \Delta_{T} q_{v}(x)=\eta x^{\alpha}(1-x)^{\beta}\left(1+\gamma x^{\delta}+\rho x\right) .
$$

For the $u$-quark distribution we find

$$
\begin{aligned}
& \eta=1.5025, \quad \alpha=1.1586, \quad \beta=3.9940 \\
& \gamma=113.65, \quad \delta=11.150, \quad \rho=12.214,
\end{aligned}
$$

and a fit to the $d$-quark distribution gives

$$
\begin{aligned}
& \eta=-2.7990, \quad \alpha=1.5952, \quad \beta=5.7531 \\
& \gamma=45.424, \quad \delta=8.0163, \quad \rho=2.2065 .
\end{aligned}
$$

These numbers produce an extremely good fit to our results, however one should not read too much into their values as a similar $\chi^{2}$ can be achieved with other sets of parameters. The transversity parametrization used in

\footnotetext{
6 The non-conservation of the nucleon tensor charge results from a non-zero anomalous dimension in Mellin space, $\Delta_{T} \gamma_{q q}(n)$, at leading order. The sign of the transversity anomalous dimension at both the leading and next-to-leading order is negative, and therefore the tensor charge drops with increasing $Q^{2}$. Moreover, since $\Delta_{T} \gamma_{q q}(n)<\Delta \gamma_{q q}(n)$ for all moments $n$, the transversity moments decrease more rapidly than the helicity moments. This has important consequences for the QCD evolution of the transversity distributions.
}

Ref. [20] has the form

$$
\begin{aligned}
x \Delta_{T} q_{v}(x)=\frac{1}{2} N_{q}^{T} & x^{\alpha}(1-x)^{\beta} \\
& \frac{(\alpha+\beta)^{(\alpha+\beta)}}{\alpha^{\alpha} \beta^{\beta}}[q(x)+\Delta q(x)],
\end{aligned}
$$

where $q(x)$ and $\Delta q(x)$ are the GRV parametrizations given in Refs. [34, 35]. Using this parametrization, a fit to our distributions at $Q^{2}=0.4 \mathrm{GeV}^{2}$ gives

$$
N_{u}^{T}=1.13, \quad N_{d}^{T}=-1.46, \quad \alpha=1.48, \quad \beta=0.70,
$$

which also furnishes a good representation of our results. These values are very different from those obtained in Ref. [20], reflecting the fact that our distributions are considerably larger.

\section{CONCLUSION}

Using a covariant quark-diquark model for the nucleon, which includes both the scalar and axial-vector diquark channels, we calculated the transversity quark distributions for the proton. A key feature of this framework is that it produces quark distributions that have the correct support and obey the number and momentum sum rules. This model also eliminates unphysical thresholds for nucleon decay into quarks and hence incorporates important aspects of confinement.

We found that at the model scale the transversity and helicity distributions are very similar in magnitude, with $\Delta_{T} u_{v}(x)$ being slightly larger than $\Delta u_{v}(x)$ for all $x$, whereas $\Delta_{T} d_{v}(x)$ becomes smaller than $\Delta d_{v}(x)$ for $x \lesssim 0.35$. These results satisfy the Soffer inequality. For the first moments of the distributions we find $\Delta_{T} u_{v}=1.04$ and $\Delta_{T} d_{v}=-0.24$, giving a nucleon isovector tensor charge of $g_{T}=1.28$ at the model scale. This result is very near the empirical value for the axial charge, $g_{A}=1.267$, which we use as a constraint to help determine the model parameters. Axial-vector diquarks were found to play a pivotal role, essentially through the scalar-axial-vector mixing term included here for the first time in a transversity calculation. This diagram almost exclusively gives rise to the $d$-quark distribution and provides about $20 \%$ of the strength for the $u$-quark transversity result. Future work in this direction will be to calculate fragmentation functions, in particular the Collins functions, so that a direct comparison between semi-inclusive DIS experiments and theory is possible.

We compared our transversity results with the recent experimental extraction of Anselmino et al., and in each case found that our distributions lie slightly outside the one-sigma confidence interval of their empirical parametrizations, in the valence quark region. The potentially small magnitude of the empirical $\Delta_{T} u(x)$ distribution, if confirmed by future experimental data, would indicate very large relativistic effects for the quarks in the 
nucleon. This would be very difficult to explain within relativistic models utilizing the concept of a constituent quark mass.

\section{Acknowledgments}

IC thanks Jefferson Lab for its hospitality and support, as this is where much of this research was com- pleted. This work was supported by: Department of Energy, Office of Nuclear Physics, contract no. DE-AC0206CH11357, under which UChicago Argonne, LLC, operates Argonne National Laboratory; contract no. DEAC05-84ER40150, under which JSA operates Jefferson Lab; and by the Grant in Aid for Scientific Research of the Japanese Ministry of Education, Culture, Sports, Science and Technology, Project no. C-19540306.
* Electronic address: icloet@anl.gov

$\dagger$ Electronic address: bentz@keyaki.cc.u-tokai.ac.jp

‡ Electronic address: awthomas@jlab.org

[1] A. J. Buras, Rev. Mod. Phys. 52, 199 (1980).

[2] M. Anselmino, A. Efremov and E. Leader, Phys. Rept. 261, 1 (1995) [Erratum-ibid. 281, 399 (1997)] [arXiv:hep-ph/9501369].

[3] V. Barone, A. Drago and P. G. Ratcliffe, Phys. Rept. 359, 1 (2002) [arXiv:hep-ph/0104283].

[4] J. C. Collins, Nucl. Phys. B 396, 161 (1993) [arXiv:hep$\mathrm{ph} / 9208213]$.

[5] R. L. Jaffe, Phys. Rev. D 54, 6581 (1996) [arXiv:hep$\mathrm{ph} / 9605456]$.

[6] J. P. Ralston and D. E. Soper, Nucl. Phys. B 152, 109 (1979).

[7] X. Artru and M. Mekhfi, Z. Phys. C 45, 669 (1990).

[8] R. L. Jaffe and X. D. Ji, Nucl. Phys. B 375, 527 (1992).

[9] R. L. Jaffe and X. D. Ji, Phys. Rev. Lett. 67, 552 (1991).

[10] A. Hayashigaki, Y. Kanazawa and Y. Koike, Phys. Rev. D 56, 7350 (1997) [arXiv:hep-ph/9707208].

[11] W. Vogelsang, Phys. Rev. D 57, 1886 (1998) [arXiv:hep$\mathrm{ph} / 9706511]$.

[12] Y. Nambu and G. Jona-Lasinio, Phys. Rev. 122, 345 (1961).

[13] Y. Nambu and G. Jona-Lasinio, Phys. Rev. 124, 246 (1961).

[14] D. Ebert, T. Feldmann and H. Reinhardt, Phys. Lett. B 388, 154 (1996).

[15] G. Hellstern, R. Alkofer and H. Reinhardt, Nucl. Phys. A 625, 697 (1997).

[16] N. Ishii, W. Bentz and K. Yazaki, Phys. Lett. B 301, 165 (1993).

[17] H. Asami, N. Ishii, W. Bentz and K. Yazaki, Phys. Rev. C 51, 3388 (1995).

[18] A. Buck, R. Alkofer and H. Reinhardt, Phys. Lett. B 286, 29 (1992).

[19] I. C. Cloët, W. Bentz and A. W. Thomas, Phys. Lett. B 621, 246 (2005) [arXiv:hep-ph/0504229].

[20] M. Anselmino, M. Boglione, U. D'Alesio, A. Kotzinian, F. Murgia, A. Prokudin and C. Turk, Phys. Rev. D 75, 054032 (2007) [arXiv:hep-ph/0701006].

[21] R. L. Jaffe, Nucl. Phys. B 229, 205 (1983).

[22] R. L. Jaffe, MIT-CTP-1261 Lectures presented at the Los Alamos School on Quark Nuclear Physics, Los Alamos, N.Mex., Jun 10-14, 1985.

[23] H. Mineo, W. Bentz and K. Yazaki, Phys. Rev. C 60,
065201 (1999) [arXiv:nucl-th/9907043].

[24] H. Mineo, W. Bentz, N. Ishii, A. W. Thomas and K. Yazaki, Nucl. Phys. A 735, 482 (2004) [arXiv:nuclth/0312097].

[25] N. Ishii, W. Bentz and K. Yazaki, Nucl. Phys. A 587, 617 (1995).

[26] H. Mineo, W. Bentz, N. Ishii and K. Yazaki, Nucl. Phys. A 703, 785 (2002) [arXiv:nucl-th/0201082].

[27] W. Bentz and A. W. Thomas, Nucl. Phys. A 696, 138 (2001).

[28] J. S. Schwinger, Phys. Rev. 82, 664 (1951).

[29] J. Bijnens, C. Bruno and E. de Rafael, Nucl. Phys. B 390, 501 (1993) [arXiv:hep-ph/9206236].

[30] A. W. Schreiber, A. I. Signal and A. W. Thomas, Phys. Rev. D 44, 2653 (1991).

[31] M. Miyama and S. Kumano, Comput. Phys. Commun. 94, 185 (1996) [arXiv:hep-ph/9508246].

[32] M. Hirai, S. Kumano and M. Miyama, Comput. Phys. Commun. 108, 38 (1998) [arXiv:hep-ph/9707220].

[33] M. Hirai, S. Kumano and M. Miyama, Comput. Phys. Commun. 111, 150 (1998) [arXiv:hep-ph/9712410].

[34] M. Gluck, E. Reya and A. Vogt, Eur. Phys. J. C 5, 461 (1998) [arXiv:hep-ph/9806404].

[35] M. Gluck, E. Reya, M. Stratmann and W. Vogelsang, Phys. Rev. D 63, 094005 (2001) [arXiv:hep-ph/0011215].

[36] S. Scopetta and V. Vento, Phys. Lett. B 424, 25 (1998) [arXiv:hep-ph/9706413].

[37] V. Barone, T. Calarco and A. Drago, Phys. Lett. B 390, 287 (1997) [arXiv:hep-ph/9605434].

[38] L. P. Gamberg, H. Reinhardt and H. Weigel, Phys. Rev. D 58, 054014 (1998) [arXiv:hep-ph/9801379].

[39] K. Suzuki and T. Shigetani, Nucl. Phys. A 626, 886 (1997) [arXiv:hep-ph/9709394].

[40] K. Suzuki and W. Weise, Nucl. Phys. A 634, 141 (1998) [arXiv:hep-ph/9711368].

[41] B. Q. Ma, I. Schmidt and J. Soffer, Phys. Lett. B 441, 461 (1998) [arXiv:hep-ph/9710247].

[42] J. Bluemlein and H. Bottcher, Nucl. Phys. B 636, 225 (2002) [arXiv:hep-ph/0203155].

[43] M. Hirai, S. Kumano and N. Saito [Asymmetry Analysis Collaboration], Phys. Rev. D 69, 054021 (2004) [arXiv:hep-ph/0312112].

[44] J. Soffer, Phys. Rev. Lett. 74, 1292 (1995) [arXiv:hepph/9409254]. 